\title{
Interstitial lung disease in patients with antisynthetase syndrome: a retrospective case series study
}

\author{
Elisa Baratella ${ }^{1} \odot$. Cristina Marrocchio ${ }^{2} \cdot$ Rossella Cifaldi $^{3} \cdot$ Mario Santagiuliana ${ }^{3} \cdot$ Alessandro Marco Bozzato $^{2}$. \\ Paola Crivelli ${ }^{4} \cdot$ Barbara Ruaro $^{3} \cdot$ Francesco Salton $^{3} \cdot$ Marco Confalonieri $^{3} \cdot$ Maria Assunta Cova $^{1}$
}

Received: 18 May 2020 / Accepted: 9 August 2020 / Published online: 2 September 2020

(c) The Author(s) 2020

\begin{abstract}
Purpose Antisynthetase syndrome (ASS) is a rare systemic autoimmune condition associated to the presence of antiaminoacyl-tRNA synthetase antibodies. Interstitial lung disease (ILD) is the most prevalent manifestation of ASS and is a major determinant of morbidity and mortality. The aim of this study was to describe the radiological characteristics of patients with ASS-associated-ILD in our institution.

Materials and methods Medical records from 2014 to 2020 were retrospectively reviewed and patients with a diagnosis of ASS and evidence of ILD on HRCT were included. HRCT images were reviewed by two thoracic radiologists in consensus. Five HRCT patterns were defined: cellular non-specific interstitial pneumonia (NSIP), organizing pneumonia (OP), mixed NSIP/OP pattern, acute interstitial pneumonia (AIP) pattern and fibrotic pattern. Descriptive statistics was calculated for all variables.

Results Twenty-two patients with ASS who met inclusion criteria were included. The disease presented with the typical triad of ASS in $45 \%$ of patients, 55\% had ILD only at the onset. Cellular NSIP was present in 27\% of patients, OP in 23\%, mixed NSIP/OP in $9 \%$, AIP in $18 \%$ and a fibrotic pattern in $23 \%$.

Conclusion HRCT findings in ASS-associated ILD are often non-specific; nevertheless, it is important to consider this diagnosis, especially in patients presenting with acute onset of symptoms.
\end{abstract}

Keywords Antisynthetase syndrome $\cdot \mathrm{HRCT} \cdot$ Acute interstitial pneumonia

\section{Introduction}

Antisynthetase syndrome (ASS) is a rare systemic autoimmune condition classified among the idiopathic inflammatory myopathies, associated to the presence of anti-aminoacyl-transfer RNA synthetase antibodies. These antibodies are directed against enzymes that attach amino acids to

Elisa Baratella

elisa.baratella@gmail.com

1 Department of Radiology, University of Trieste, Strada di Fiume 447, 34128 Trieste, Italy

2 Department of Medicine, Surgery and Health Science, University of Trieste, Strada di Fiume 447, Trieste, Italy

3 Department of Pneumology, Azienda Sanitaria Universitaria Giuliano Isontina (ASUGI), Strada di Fiume 447, Trieste, Italy

4 Diagnostic Imaging 2, AOU Sassari, viale S. Pietro 43, Sassari, Italy their cognate transfer ribonucleic acid during polypeptide synthesis. At present, eight antisynthetase antibodies have been identified, the most frequent being anti-Jo1 (histidyl), followed by anti-EJ (glycyl), anti-PL7 (threonyl) and antiPL12 (alanyl) [1]. ASS is characterized by a classical triad of Interstitial Lung Disease (ILD), myositis, and positivity to antisynthetase antibodies and other less specific symptoms such as polyarthritis, mechanic's hands, Raynaud's phenomenon, or symptom overlapping with Sjogren syndrome and systemic sclerosis [2].

Interstitial lung disease (ILD) is the most prevalent manifestation of ASS, occurring in 71-100\% patients, and may be the only clinical manifestation at the onset of the disease $[2,3]$. ILD is a major determinant of morbidity and mortality in antisynthetase syndrome, and the extension of lung involvement correlates with the prognosis of the disease [4]. High-resolution computed tomography (HRCT) plays a main role in ASS in diagnosing ILD, in identifying the pattern of involvement at the onset of symptoms and in assessing the 
resolution, stability or progression of the pulmonary alterations during follow-up [5-7].

The most common radiological patterns described in ASS-associated ILD are non-specific interstitial pneumonia (NSIP) and organizing pneumonia (OP). Less commonly, usual interstitial pneumonia (UIP) and acute interstitial pneumonia (AIP) have been reported. Nevertheless, the evidence is currently based on retrospective studies or case reports [8-10]. In our clinical practice, we often encountered patients finally diagnosed with ASS who had HRCT findings reported to be less frequent in this syndrome. It is important to be aware of all the possible ASS presentations on HRCT, to consider this syndrome among the differential diagnoses even in atypical cases, avoiding delays in the diagnosis.

In this study we report our experience describing the clinical characteristics and the HRCT radiological findings of a series of patients with a diagnosis of ASS in the last five years at our institution.

\section{Materials and methods}

The study was approved by the local internal ethical review board and has been performed in accordance with the ethical standards laid down in the Declaration of Helsinki. All patients gave informed consent prior to their inclusion in the study. Medical records in our Institution from 2014 to 2020 were retrospectively reviewed and patients were included if they met the following inclusion criteria: (i) 18 years or older; (ii) the diagnosis of antisynthetase syndrome; (iii) HRCT performed since the diagnosis with evidence of ILD; (iv) no other diseases presenting with abnormal findings on chest CT (e.g. lung cancer, pulmonary infections, sarcoidosis). Patients were diagnosed with ASS syndrome according to the criteria proposed by Solomon et al. [11], after multidisciplinary consultation involving at least a thoracic radiologist, an expert rheumatologist, a pneumologist and a pathologist. Demographics and relevant clinical data including disease presentation and symptoms at diagnosis were registered. HRCT was performed with 256-row multidetector CT system (Brilliance iCT 256, Philips, Best, The Netherlands) and acquired during single breath hold at full inspiration, with the patient in a supine position. Technical parameters were as follows: rotation time, $270 \mathrm{~ms}$; beam collimation, $128 \times 2 \times 0.625 \mathrm{~mm}$; normalized pitch, 0.975 ; $z$-axis coverage, $160 \mathrm{~mm}$; reconstruction interval, $0.3 \mathrm{~mm}$; section reconstruction thickness, $1 \mathrm{~mm}$; tube voltage, $120 \mathrm{kV}$; tube current (effective mA), 280-400 depending on patient size; and field of view, $40 \mathrm{~cm}$. CT images were analyzed at standard lung window settings (window level of $-600 \mathrm{HU}$ and window width of $2000 \mathrm{HU}$ ) and mediastinal window setting (window level 400-500 HU and window width 20-40 HU).
Images were reviewed in consensus by two thoracic radiologists with 15 and 10 years of experience, who were aware that only patients with ASS were included but were blinded to clinical symptoms and other patients' characteristics. Discrepant interpretations were resolved by consensus through the involvement of a third reader with 20 years of experience.

The following CT findings were evaluated: reticulations, traction bronchiectasis, honeycombing, ground glass opacities (GGO), air-space consolidations. According to the Fleischner society recommendation, reticulations were inter- and intra-lobular septal thickening; traction bronchiectasis was defined as bronchial and bronchiolar irregular dilatation caused by fibrotic changes of the surrounding lung parenchyma; honeycombing presented as clustered cystic air spaces with thick and well-defined walls, usually in dorsal and subpleural regions of the chest; GGO was defined as a hazy increased lung attenuation that does not obscure the bronchovascular structures; air-space consolidation was a homogeneous increase in pulmonary parenchymal attenuation [12].

Based on CT findings, five HRCT patterns were defined: cellular non-specific interstitial pneumonia (NSIP), organizing pneumonia (OP), mixed NSIP/OP pattern, acute interstitial pneumonia pattern and fibrotic pattern [5]. According to the ATS/ERS classification of idiopathic interstitial pneumonia, cellular NSIP was characterized by GGO generally bilateral, symmetrical and with a prevalently medial and basal distribution [5]. Fibrotic NSIP, characterized by irregular septal thickening and signs of fibrosis such as traction bronchiectasis and architectural distortion, was included in the fibrotic pattern. OP presented with air-space consolidations in a typically subpleural and peribronchial distribution and with migratory behavior. The pattern was considered mixed NSIP/OP when consolidations were superimposed on a background of GGO [13]. In case of acute presentation, within a month, acute interstitial pneumonia (AIP pattern) was reported if bilateral patchy GGO were present, often associated with areas of consolidation. When signs of lung fibrosis (honeycombing, traction bronchiectasis, irregular septal thickening and reticulations) were present, the pattern was classified as fibrotic. In this category, the definite and probable UIP and the fibrotic NSIP patterns were included. UIP was reported according to the ATS/ERS/JRS/ALAT guidelines as definite, when honeycombing and reticular opacities were present in a basal and peripheral distribution, often with traction bronchiectasis, and as probable when the honeycombing was missing [5]. The CT information was completed by all patients' clinical characteristics.

Other signs of chest involvement were noted, including pleural effusion, pericardial effusion, and presence of pulmonary hypertension. Pulmonary hypertension was reported when the main pulmonary artery had a diameter of $29 \mathrm{~mm}$ 
or more or the ratio of the diameter of the main pulmonary artery to the diameter of the ascending aorta was greater than 1:1. Indirect signs of pulmonary hypertension were considered to be presence of right heart disease, such as right ventricular enlargement and hypertrophy, or suggestive parenchymal features, including mosaic attenuation.

Descriptive statistics was calculated for all variables. Results are expressed as mean $\pm \mathrm{SD}$; frequencies and proportions are provided for categorical variables.

\section{Results}

Twenty-two patients with ASS who met inclusion criteria were included. Sixteen patients were female (73\%) and six were males $(27 \%)$ with a mean age at diagnosis of $52 \pm 6$ years. Ten patients $(45 \%)$ presented with the typical triad of ILD, myositis, and serum positivity for anti-aminoacyl-tRNA synthetase antibodies, whereas the remaining patients $(12 / 22,55 \%)$ had only ILD as clinical manifestation at diagnosis. Signs and symptoms of pulmonary involvement were dyspnea in $19(86 \%)$ and cough in 17 patients (77\%); four patients (18\%) presented with acute respiratory failure. At physical examination we observed skin signs, i.e. erythema, in only one patient.

Regarding antisynthetase antibodies, anti-Jo-1 was detected in six patients, anti-OJ in one, anti-PL-12 in one, anti-PL-7 in one and anti-EJ in one patient at admission. Antibodies of collagen vascular diseases were also evaluated; in particular, anti-SSA were present in three patients, anti-SSB in one patient, antinuclear antibodies (ANF) were present in five patients, and anti-RF were present in one patient i.e. anti-neutrophil cytoplasmic antibodies. No other autoantibodies were observed. An increased serum level of myogenic enzyme was observed in 4 patients.

Cellular NSIP or OP pattern were the presenting patterns in $50 \%$ of patients, specifically $6(27 \%)$ had cellular NSIP (Fig. 1), 5 (23\%) OP (Figs. 2, 3), and 2 (9\%) had a mixed NSIP/OP pattern (Fig. 4). The HRCT of the 4 patients (18\%) who presented with acute respiratory failure showed an AIP pattern, with extensive and inhomogeneous ground glass opacities, areas of consolidations and sparing of nondependent regions of the lungs (Fig. 5). Fibrotic changes were present in 5 patients (23\%) with honeycombing, traction bronchiectasis and reticular opacities (Figs. 6, 7, 8). Specifically, 2 patients were diagnosed with definite UIP, 1 patient with probable UIP and 2 patients with fibrotic NSIP.

Pleural effusion was present in 9 of the twenty-two patients (41\%) and pulmonary hypertension in 6 cases (27\%) (Fig. 9). Pericardial effusion was a less common finding, occurring in only 3 patients.

\section{Discussion}

The present study reports the clinical and radiological characteristics of a series of twenty-two patients with a diagnosis of antisynthetase syndrome and evidence of interstitial lung disease on HRCT.

ASS is a rare systemic autoimmune disease diagnosed in the presence of antisynthetase antibodies and compatible clinical features, in particular ILD and/or inflammatory myositis. Because of heterogeneity in the observed phenotype and severity of the disease, diagnosis of ASS remains difficult. The clinical and demographic findings in our patients were in line with those reported in ASS patients. The disease was more prevalent in middle-aged women and
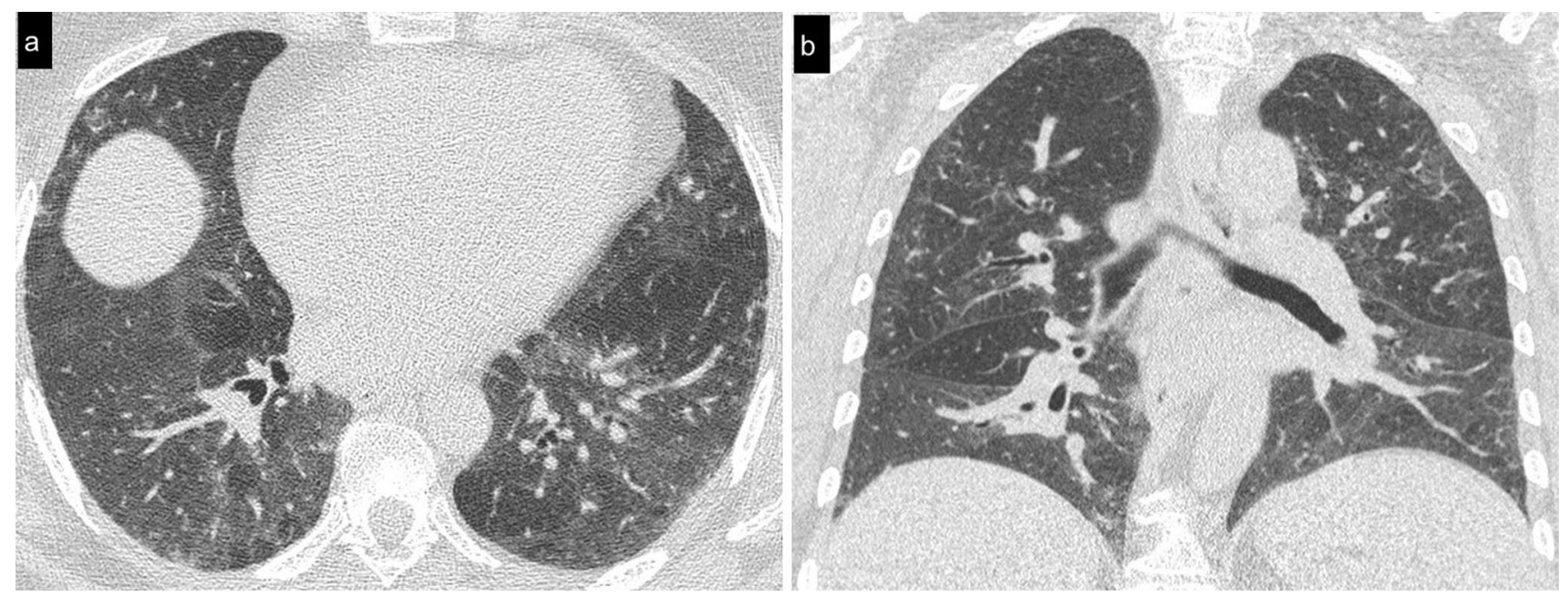

Fig. 1 Cellular NSIP pattern: the multiplanar axial (a) and coronal (b) reconstructions show diffuse areas of ground glass opacities without traction bronchiectasis 

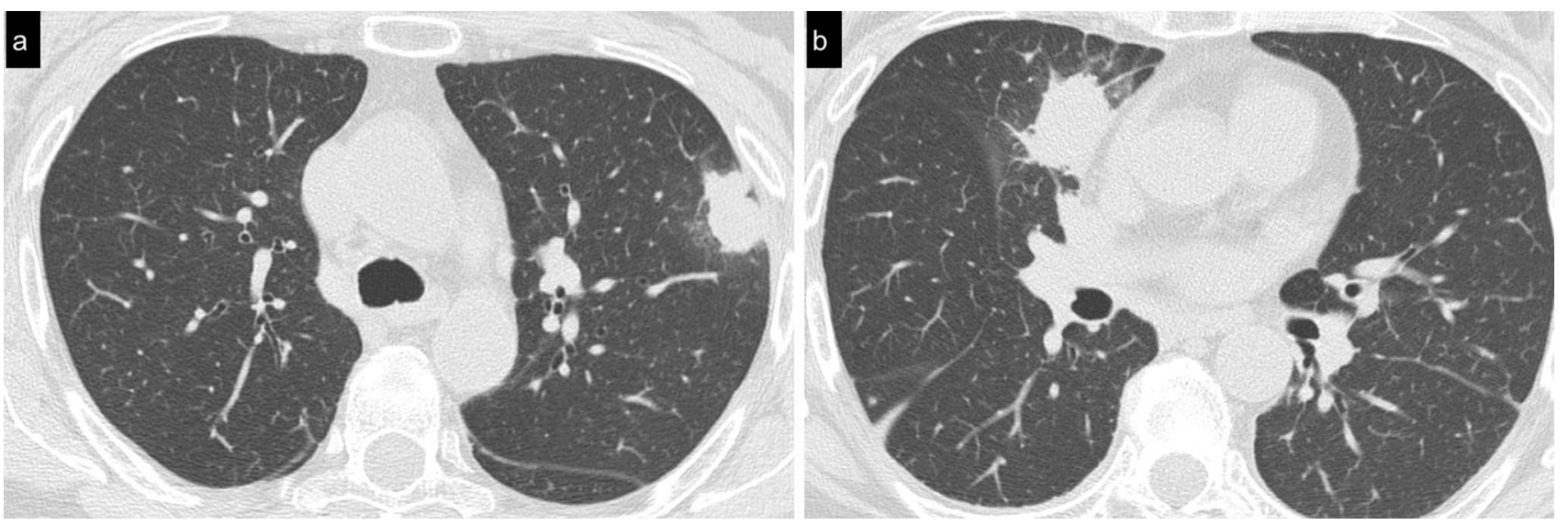

Fig. 2 Organizing pneumonia pattern: air-space consolidations surrounded by a ground glass halo were present bilaterally

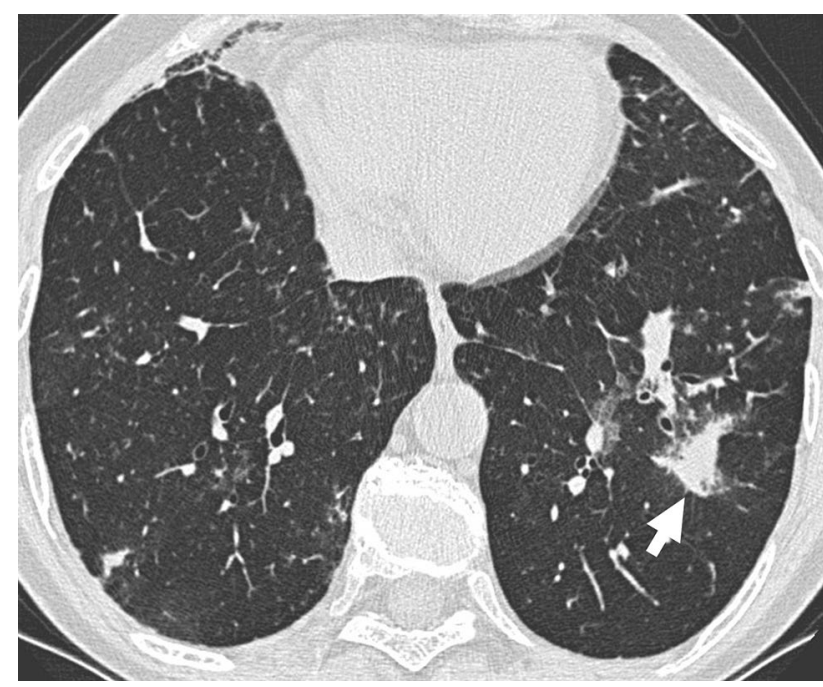

Fig. 3 Organizing pneumonia pattern: air-space consolidation with a peribronchial distribution (arrow) is present in the lower left lobe in $45 \%$ of cases it presented with the classic triad of ILD, myositis and presence of antisynthetase antibodies. In 55\% of cases, ILD was the only clinical presentation at the onset of the disease, and this underlines the importance of HRCT evaluation and correct interpretation in these patients [2, 14]. Current evidence on HRCT patterns in ASS-associated ILD is based predominantly on retrospective case series and case reports. NSIP has been reported as the most common pattern on HRCT in patients with ILD secondary to autoimmune etiology, in particular in patients with antisynthetase syndrome, followed by OP. Less common is the occurrence of usual interstitial pneumonia [8-10]. In our case series, only half of the patients presented with cellular NSIP and OP patterns. Fibrotic changes in the lung parenchyma were found with the same prevalence as OP. In previous reports, the UIP pattern has been reported to be associated with a worse prognosis [15].
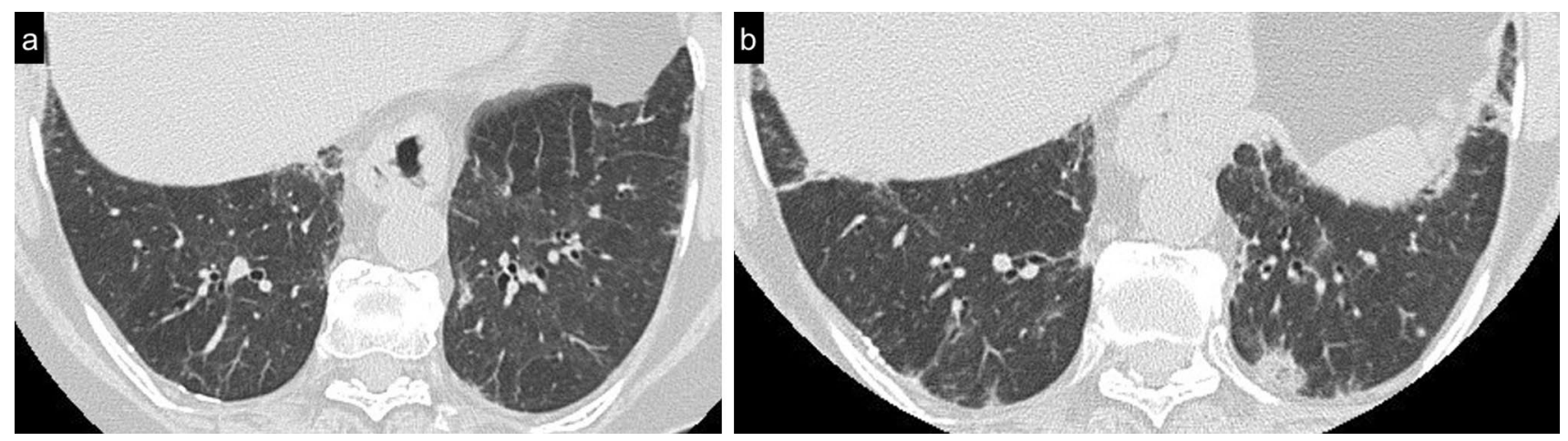

Fig. 4 Mixed NSIP/OP pattern: axial CT images of the same patient at different levels show consolidations superimposed on a background of ground glass opacities 

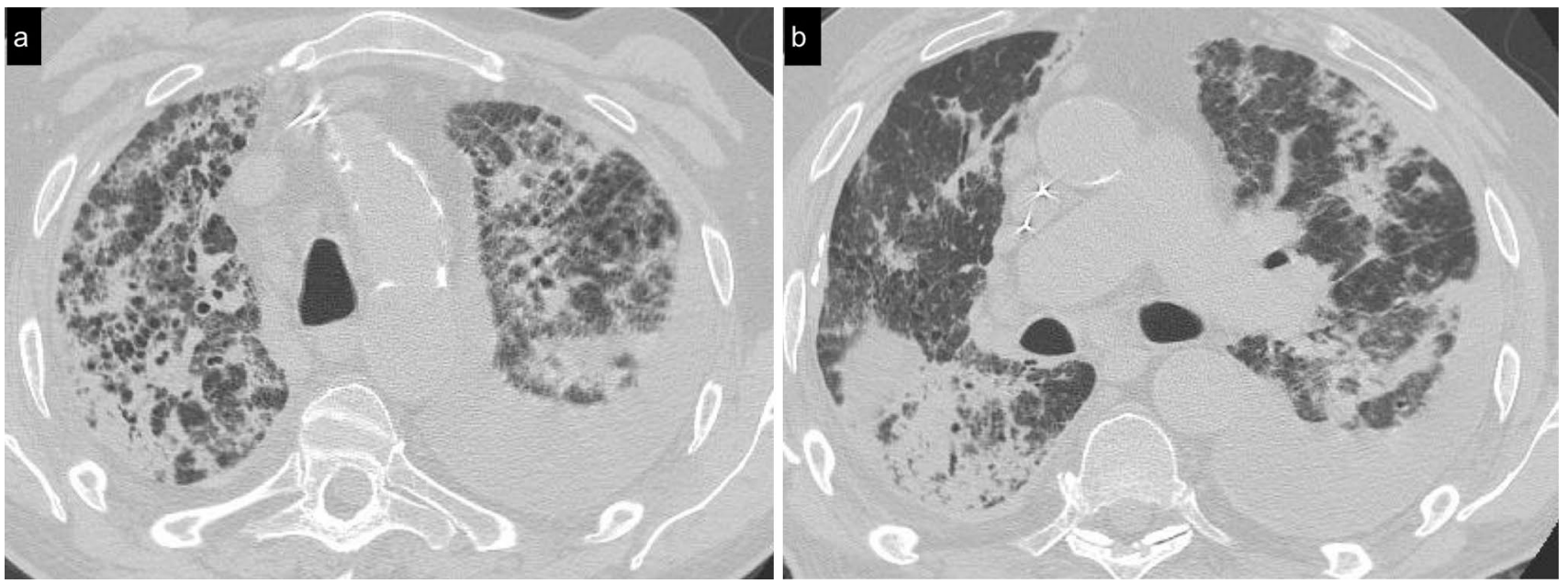

Fig. 5 Acute interstitial pneumonia pattern: bilateral inhomogeneous patchy ground glass opacities with areas of consolidation, non-dependent areas of sparing and some traction bronchiectasis are present

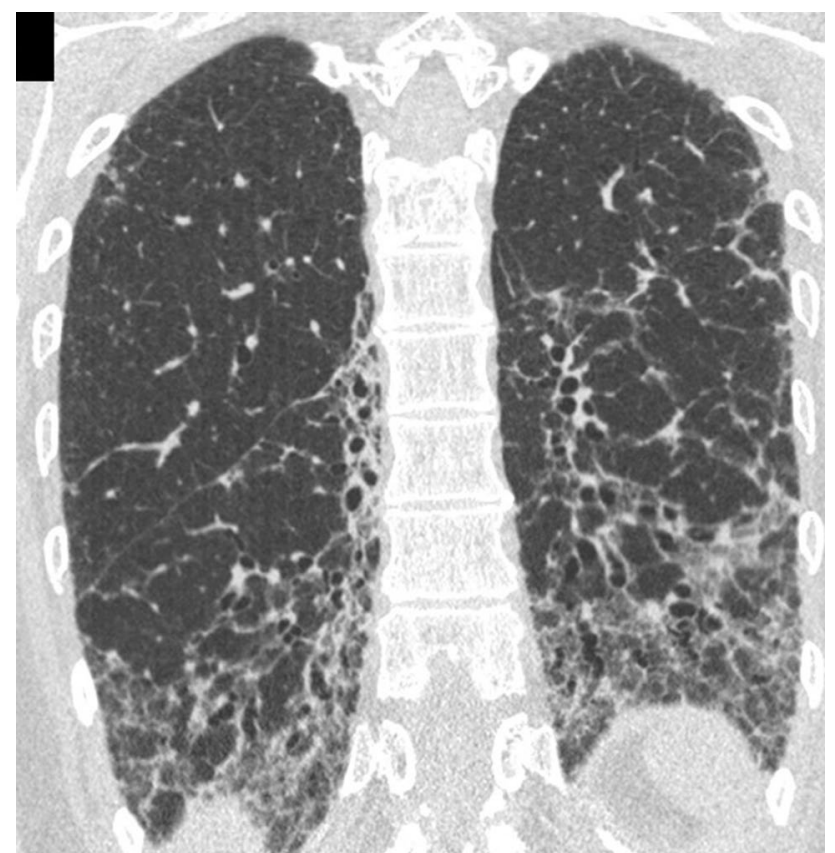

Fig. 6 Fibrotic NSIP pattern. The multiplanar coronal reconstruction of this CT scan demonstrates extensive bilateral ground glass opacities with traction bronchiectasis with a peripheral and basal distribution

An interesting finding of this study is that ASS had an acute presentation with acute interstitial pneumonia in 4 of 22 patients, accounting for almost $20 \%$ of cases. Acute respiratory failure is an uncommon presentation of ASS and may be an unsuspected cause of ARDS, especially if no other extra-pulmonary symptoms of the disease are present. The typical HRCT finding in these patients is an acute interstitial pneumonia pattern, with bilateral patchy GGO often associated to areas of consolidations, and this was the presentation in all our patients with acute onset. Identifying the cause of the acute respiratory failure is a crucial step for initiating a targeted treatment and improving diagnosis, therefore ASS should be considered among the possible causes and, in cases in which it is suspected, immunological tests should be included in the diagnostic work-up [16].

Chest findings in antisynthetase syndrome are not limited to the lung parenchyma and the other manifestations of the disease should always be noted, especially pulmonary hypertension. Although pleural effusion is quite a common finding in patients with connective tissue diseases, in particular in rheumatoid arthritis and systemic lupus erythematosus, it is not frequent in patients with ASS [17]. Only few cases have been reported of ASS-associated pericardial effusion, and indeed this finding was present in only 3 cases in our experience [18].

The radiological characteristics of ASS are often nonspecific. The differential diagnosis is broad and has to be made with other main causes of ILD, both idiopathic and secondary, including collagen vascular diseases, drug toxicity, hypersensitivity pneumonitis and asbestosis [19]. To reach the final diagnosis, a multidisciplinary evaluation is highly recommended to evaluate the clinical, serological and radiological findings in each patient $[6,20]$.

Our study has some limitations. This is a case series with a relatively small sample size, which is a limitation inherent to the rarity of the disease. Moreover, due to its retrospective nature, patients had heterogeneous disease characteristics and not uniform timing of HRCT examinations. Nevertheless, about $50 \%$ of our patients presented with findings reported to be less common in ASS. This should improve the 


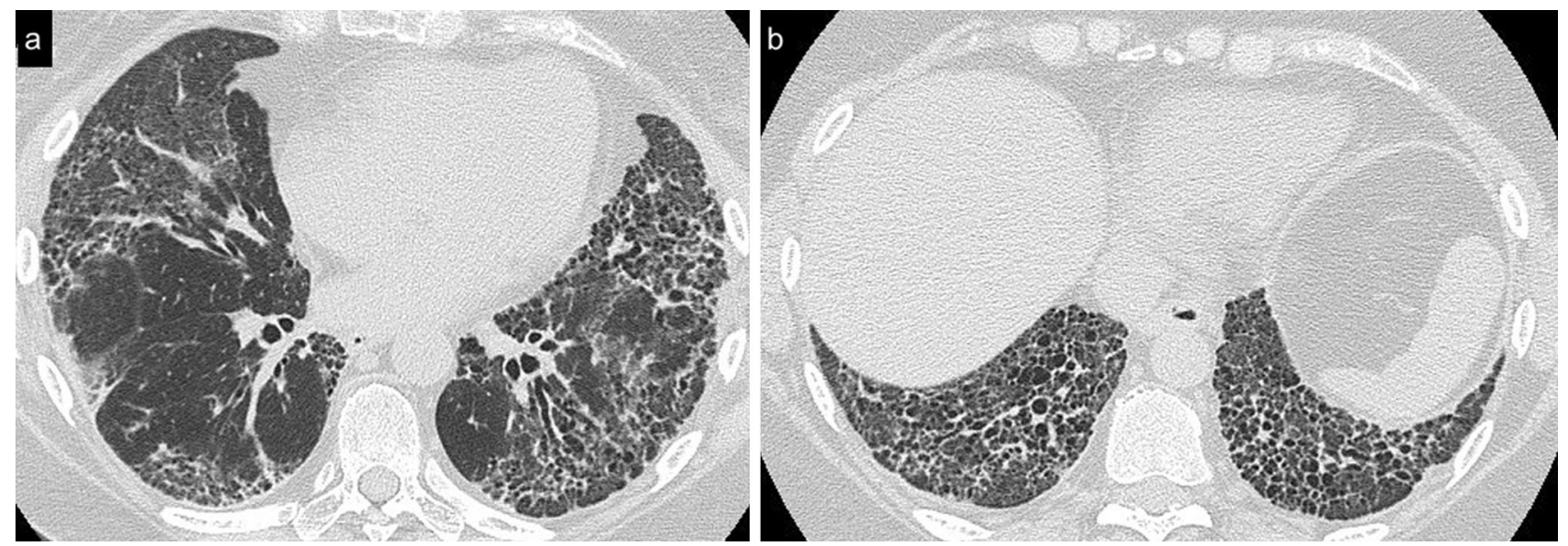

Fig. 7 Typical usual interstitial pneumonia (UIP) pattern. Multiplanar axial reconstructions at different levels show an irregular inter- and intra-lobular septal thickening, with a subpleural, basal predomi- nance, traction bronchiectasis and bronchiolectasis (a) and honeycombing (b), which is clearly seen in the basal region
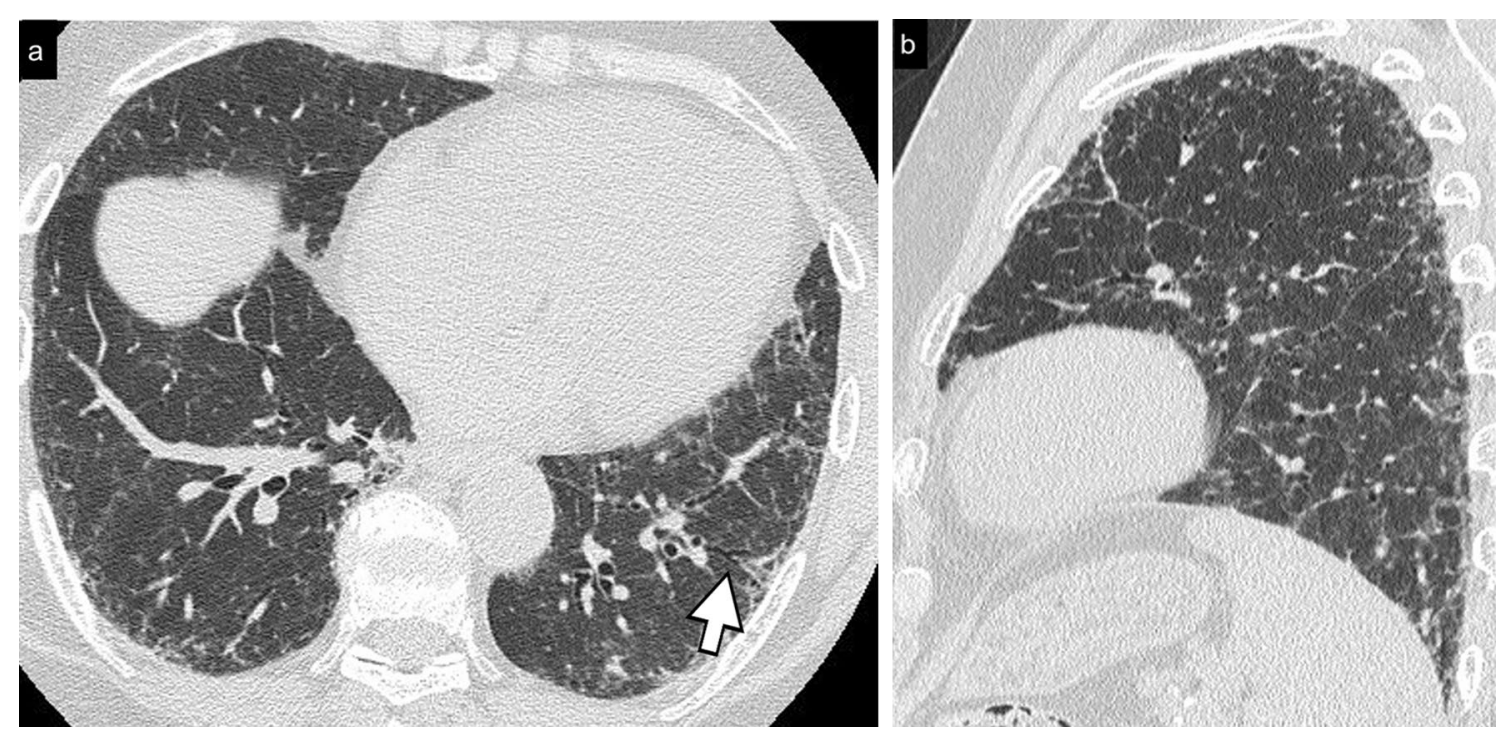

Fig. 8 Probable UIP pattern, axial (a) and sagittal (b) multiplanar reconstructions: irregular inter- and intra-lobular septal thickening, with a subpleural, basal predominance. Traction bronchiectasis and bronchiolectasis are seen in the fibrotic areas (arrow); irregular thickening of the pleural surface is better visualized in the sagittal plane. Honeycombing is not present awareness of the wide spectrum of HRCT findings in ASS to consider this disease in the differential diagnosis also in challenging cases and especially in those patients presenting with an acute onset and no extra-pulmonary manifestations of the disease.

\section{Conclusion}

HRCT patterns in ASS-associated ILD are often non-specific, but it is important to maintain a high suspicion especially in those patients presenting with acute respiratory failure. 


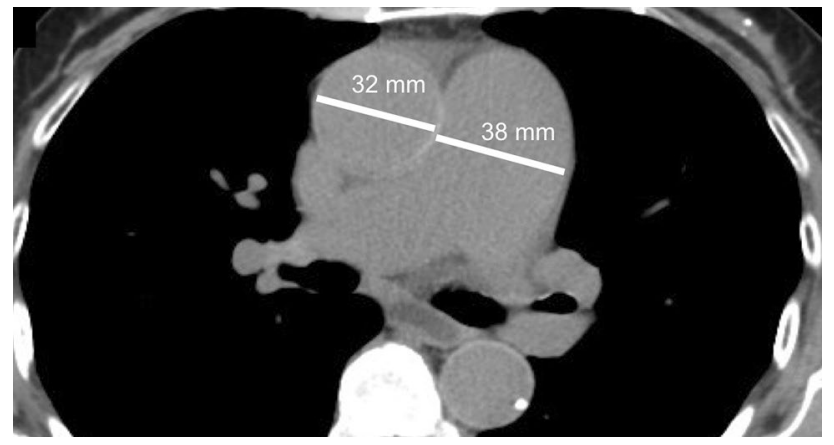

Fig. 9 Pulmonary hypertension: the main pulmonary arterial diameter measured at the level of its bifurcation on the axial CT scan is $38 \mathrm{~mm}$ and the ratio of the diameter of the main pulmonary artery to the diameter of the ascending aorta is greater than 1:1

Funding Open access funding provided by Università degli Studi di Trieste within the CRUI-CARE Agreement. No specific funding was received from any bodies in the public, commercial or not-for-profit sectors to carry out the work described in this article.

\section{Compliance with ethical standards}

Conflict of interest The authors declare that they have no conflict of interest.

Ethical approval All procedures performed in studies involving human participants were in accordance with the ethical standards of the institutional and/or national research committee and with the 1964 Helsinki declaration and its later amendments or comparable ethical standards.

Open Access This article is licensed under a Creative Commons Attribution 4.0 International License, which permits use, sharing, adaptation, distribution and reproduction in any medium or format, as long as you give appropriate credit to the original author(s) and the source, provide a link to the Creative Commons licence, and indicate if changes were made. The images or other third party material in this article are included in the article's Creative Commons licence, unless indicated otherwise in a credit line to the material. If material is not included in the article's Creative Commons licence and your intended use is not permitted by statutory regulation or exceeds the permitted use, you will need to obtain permission directly from the copyright holder. To view a copy of this licence, visit http://creativecommons.org/licenses/by/4.0/.

\section{References}

1. Ghirardello A, Doria A. New insights in myositis-specific autoantibodies. Curr Opin Rheumatol. 2018;30(6):614-22.

2. Hervier B, Benveniste O. Clinical heterogeneity and outcomes of antisynthetase syndrome. Curr Rheumatol Rep. 2013;15(8):349.

3. Hamaguchi Y, Fujimoto M, Matsushita T, Kaji K, Komura K, Hasegawa M, et al. Common and distinct clinical features in adult patients with anti-aminoacyl-tRNA synthetase antibodies: heterogeneity within the syndrome. PLoS ONE. 2013;8(4):e60442.

4. Rojas-Serrano J, Herrera-Bringas D, Mejía M, Rivero H, MateosToledo H, Figueroa JE. Prognostic factors in a cohort of antisynthetase syndrome (ASS): serologic profile is associated with mortality in patients with interstitial lung disease (ILD). Clin Rheumatol. 2015;34(9):1563-9.

5. Raghu G, Remy-Jardin M, Myers JL, Richeldi L, Ryerson CJ, Lederer DJ, et al. Diagnosis of idiopathic pulmonary fibrosis. An official ATS/ERS/JRS/ALAT clinical practice guideline. Am J Respir Crit Care Med. 2018;198(5):e44-e68.

6. Lynch DA, Sverzellati N, Travis WD, Brown KK, Colby TV, Galvin JR, et al. Diagnostic criteria for idiopathic pulmonary fibrosis: a Fleischner Society White Paper. Lancet Respir Med. 2018;6(2):138-53.

7. Katzap E, Barilla-LaBarca M-L, Marder G. Antisynthetase syndrome. Curr Rheumatol Rep. 2011;13(3):175-81.

8. Gasparotto M, Gatto M, Saccon F, Ghirardello A, Iaccarino L, Doria A. Pulmonary involvement in antisynthetase syndrome. Curr Opin Rheumatol. 2019;31(6):603-10.

9. Waseda Y, Johkoh T, Egashira R, Sumikawa H, Saeki K, Watanabe $\mathrm{S}$, et al. Antisynthetase syndrome: pulmonary computed tomography findings of adult patients with antibodies to aminoacyl-tRNA synthetases. Eur J Radiol. 2016;85(8):1421-6.

10. Debray M-P, Borie R, Revel M-P, Naccache J-M, Khalil A, Toper $\mathrm{C}$, et al. Interstitial lung disease in anti-synthetase syndrome: initial and follow-up CT findings. Eur J Radiol. 2015;84(3):516-23.

11. Solomon J, Swigris JJ, Brown KK. Doença pulmonar intersticial relacionada a miosite e a síndrome antissintetase. J Bras Pneumol. 2011;37(1):100-9.

12. Hansell DM, Bankier AA, MacMahon H, McLoud TC, Müller NL, Remy J. Fleischner society: glossary of terms for thoracic imaging. Radiology. 2008;246(3):697-722.

13. Fischer A, Swigris JJ, du Bois RM, Lynch DA, Downey GP, Cosgrove GP, et al. Anti-synthetase syndrome in ANA and anti-Jo-1 negative patients presenting with idiopathic interstitial pneumonia. Respir Med. 2009;103(11):1719-24.

14. Cavagna L, Nuño L, Scirè CA, Govoni M, Longo FJL, et al. Serum Jo-1 autoantibody and isolated arthritis in the antisynthetase syndrome: review of the literature and report of the experience of AENEAS collaborative group. Clin Rev Allerg Immunol. 2017;52(1):71-80.

15. Marie I, Josse S, Hatron PY, Dominique S, Hachulla E, Janvresse $\mathrm{A}$, et al. Interstitial lung disease in anti-Jo-1 patients with antisynthetase syndrome: ILD in anti-Jo-1 patients. Arthritis Care Res. 2013;65(5):800-8.

16. Vuillard C, de Chambrun MP, de Prost N, Guérin C, Schmidt M, Dargent A, et al. Clinical features and outcome of patients with acute respiratory failure revealing anti-synthetase or anti-MDA-5 dermato-pulmonary syndrome: a French multicenter retrospective study. Ann Intensive Care. 2018;8(1):87.

17. Bouros D, Pneumatikos I, Tzouvelekis A. Pleural involvement in systemic autoimmune disorders. Respiration. 2008;75(4):361-71.

18. Maturu V, Lakshman A, Bal A, Dhir V, Sharma A, Garg M, et al. Antisynthetase syndrome: an under-recognized cause of interstitial lung disease. Lung India. 2016;33(1):20.

19. Wuyts WA, Cavazza A, Rossi G, Bonella F, Sverzellati N, Spagnolo P. Differential diagnosis of usual interstitial pneumonia: when is it truly idiopathic? Eur Respir Rev. 2014;23(133):308-19.

20. Chartrand S, Swigris JJ, Peykova L, Chung J, Fischer A. A multidisciplinary evaluation helps identify the antisynthetase syndrome in patients presenting as idiopathic interstitial pneumonia. J Rheumatol. 2016;43(5):887-92.

Publisher's Note Springer Nature remains neutral with regard to jurisdictional claims in published maps and institutional affiliations. 